\title{
Evolution of legionella control in France 1998-2018
}

\author{
Hartemann Philippe* \\ School of Medicine of Nancy, University of Lorraine, 7 Avenue de la forêt de Haye, France
}

\begin{abstract}
Due to mediatic outbreaks occurred in France at the end of the 90's, a national proactive strategy for the control of Legionella both in the cooling towers and hot water distribution networks in public buildings has been defined. Regulations and guidelines were put into force from 1999 until recently, covering other possible reservoirs, including active surveillance of clinical cases, mandatory sampling and quantitative numerations of Legionella pneumophila, control measures and reporting. The results described in this article present the epidemiological situation and the actual decrease of the number of clinical cases, illustrating the efficacy of this strategy and the disappearance of outbreaks despite numerous remaining isolated cases without reported origin.
\end{abstract}

\section{Introduction}

After the discovery of Legionella pneumophila in the US, some initiatives against the infections due to this bacterium have been taken in France but the initial shock occurred during year 1998. This was during the world football championship, organized in France, where the national team defeated the Brazilian one 3-0 during a memorable final play in Paris. In the same time a Legionellosis outbreak occurred, involving some foreign supporters who were diagnosed after their return at home, leading to an European alert via the EWGLI network. The epidemiological study performed retrospectively was able to identify the source of contamination in a Cooling Tower (CT) located in a very touristic street of Paris [1]. This strain is now known as Legionella pneumophila Paris.

The French Ministry of Environment decided to make mandatory the notification and the microbiological control of CTs. In the same time the Ministry of Health decided to organize compulsorily an active surveillance of legionellosis cases and microbiological controls of water networks in Health Care settings.

In September 2000 occurred the opening ceremony of the European Hospital Georges Pompidou in Paris, considered at this time as the most modern hospital of the country. Three months later appeared 11 cases of legionellosis (5 fatal issues) from December 2000 to June 2001 [2]. This outbreak turned into a « scandal » because some defects were evidenced in the hot water distribution network like stagnation, inadequate temperature, inadequate piping materials, etc. For the first time, point of use filters (porosity 0.22 micrometer) were largely used in patients' rooms during the necessary period for the restructuration of both hot and cold water distribution networks. The decision of the court was to pinpoint characterized faults and decide manslaughter through negligence. The consequences of this outbreak were an intense pressure of the media and patients associations, high costs for remediation and the decision of the minister to organize an adequate control inside hospitals and public buildings for " never seeing this again $»$ ! Thus, a working group in charge to propose guidelines and adequate solutions for avoiding the reproduction of such situations was created in 2001.

\section{Regulations for controlling exposure to Legionella-lad- en aerosols.}

The first regulation was dedicated in 1999 to C.Ts with a mandatory declaration of their existence to the local authorities and a monthly surveillance of the results of the maintenance by the maesurement of Legionella spp concentration in the water flow. According to the size of the CT, the owner must report to the authorities or keep these results for an eventual inspection. Two concentrations levels were determined: 1,000 CFU Legionella/L considered as a target and 1,000,000 CFU Legionella/L as an unacceptable level inducing the shutdown of the CT and mandatory operations of disinfection and adequate maintenance with new satisfactory results before restarting the CT. Because of the paucity of legionellosis cases not due to Legionella pneumophila, it has been later decided to take into consideration only this species. This has also leaded to a drastic decrease of the unnecessary disinfections.

Concurrently a dedicated team in the "Institut de Veille Sanitaire» was in charge to manage the active surveillance of all legionellosis clinical cases ad to publish the results yearly in the «Bulletin Epidémiologique Hebdomadaire» (weekly bulletin). Clear and rapid progresses were made in this surveillance, leading France to have the higher incidence rate in Europe (2.5 cases/100,000 inhabitants in 2005 [3]. The results of this surveillance are presented in the figure 1.

The yearly evolution of the number of notified cases is characterized by a rapid increase from 1996 to 2005, due to the sensitisation of MDs and hospitals with a systematic practice of urinary antigen detection in front of severe pulmonary symptoms. It is important to highlight that the French incidence of legionellosis is still the highest in Europe, probably illustrating a good notification rate. Then the number of cases begins to diminish with two unexplained peaks in 2010 and 2017 probably due to climatic events (very hot summers), in absence of

*Correspondence to: Hartemann philippe, School of Medicine of Nancy, University of Lorraine, 7 Avenue de la forêt de Haye, 545àà Vandoeuvre-Nancy, France, E-mail : philippe.hartemann@univ-lorraine.fr

Received: February 18, 2019; Accepted: February 22, 2019; Published: February 27, 2019 
important outbreaks since more than 10 years. The largest outbreak occurred during the winter $2003 / 2004$ in the Lens region, involving 86 confirmed cases, due to a petrochemical factory with an aerated basin in a wastewater treatment plant and CTs [4]. It is also remarkable to see the differences of the incidence rate according to the geographical location of the regions in a context of similar regulations for both the survey of water networks and CTs and laboratory analyses. We may note an increasing gradient from the western to the eastern regions, in accordance with dominating wind's direction.

Despite active surveillance, mandatory questionnaire and water analyses for each case, more than $50 \%$ of these cases remain unexplained in term of origin of the contamination.

It is important to make a focus on the evolution of the number of cases present in a Health Care Settings during their incubation period. The following table 1 presents the evolution of the percentage of these cases during this period of active surveillance and proactive approach with quantitative limit values and mandatory control for $L$. pneumophila in the hot water distribution network inside buildings.

The percentage of nosocomial cases has decreased dramatically during this period from $20 \%$ to around 5 to $6 \%$ during the recent years. We may hypothesize this is linked to the proactive strategy in place in each hospital and it is also important to note the absence of outbreak; they are now only single cases or small clusters of a very few cases. We may also underline that the percentage of these cases in comparison to the total of explained cases is also decreasing from 33\% in 1998 to now around $20 \%$.

This has been obtained through the application of technical measures for a better design and maintenance of internal water distribution networks and the mandatory control of Legionella pneumophila concentrations in the different types of water used for the cares exposing the patient to a risk of aerosol inhalation. This has been published in 2005 by the French Ministry of Health [5] and described in a previous article [6]. Following an attempt of quantitative risk assessment, it has been decided by the French National Council of Hygiene in 2001 to prescribe a target value of 1,000 CFU Legionella pneumophila/L in hot water at the point of use, because of an estimated risk around 1 case per one million exposed patients. This allows to avoid outbreaks but not isolated cases. Thus, a lower alert level for immunocompromised and immunosuppressed patients, under the level of quantification of the analytical method, has been recommended, in application of the precautionary principle as prescribed in the French Constitution. These values are also the alert level driving to an intensified surveillance of the network, including information, reinforcement of analytical sampling and technical measures for avoiding Legionella proliferation. A maximum admissible level of 10,000 CFU/L induces mandatorily to stop any patient's exposure.

For obtaining these levels, a good design and maintenance of the network with a temperature of the distributed hot water in a loop between 60 and $55^{\circ} \mathrm{C}$ is normally sufficient. For the immunocompromised patients, point of use filters are used both for the tap and the shower head in their room and all technical premises.

In the figure 2 are presented the most mandatory important steps for the hot water distribution network in public buildings, especially hospitals and nursing homes.

The same guidelines published in 2005 impose to fulfil the analytical enumeration's values presented in the table 2 which is summarizing the different levels according to the classification in green, orange and
Table 1. Evolution of the legionellosis cases in hospitals in France 1998- 2013

\begin{tabular}{|c|c|c|c|}
\hline Year & Total cases & Hospital cases & Percentage \\
\hline 1998 & 610 & 119 & 20 \\
\hline 2000 & 807 & 105 & 13 \\
\hline 2002 & 1044 & 89 & 9 \\
\hline 2004 & 1220 & 93 & 8.5 \\
\hline 2006 & 1443 & 87 & 6 \\
\hline 2008 & 1244 & 83 & 7 \\
\hline 2010 & 1540 & 110 & 7 \\
\hline 2013 & 1262 & 86 & 6 \\
\hline
\end{tabular}

Table 2. The different limit values used in France for evaluating the quality of hot water inside hospitals

\section{Legionella pneumophila control in France in hot water}

- HOSPITAL + PUBLIC BUILDINGS

- taRGet VALUE $:<1,000$ UFC LP/L

- alert VAlUe : $>1,000$ UFC Lp/L

(information) confirmation - diagnosis - plumbing works

- maximal Value : 10,000 UfC Lp/L

Information - restriction of use - remediation - reorganization - control

- HOSPITAL IMMUNOCOMPROMIZED PATIENTS

- TARGET VALUE : ABSENCE of Legionella

- alert VAlue : (50) 250 UfC lp/L

- maximal VAlUe : (50) 250 UFC Lp/L

CONSEQUENCE: NO MORE LEGIONELLOSIS HOSPITAL OUTBREAK IN FRANCE !!

red (target, alert, unacceptable levels). The full respect of these values needed some years to be everywhere achieved in the French healthcare settings, because of the requested changes in the plumbing system and the induced costs. But it is now clear the situation is in accordance with these guidelines since the 2010's, with the simultaneous disappearance of legionellosis outbreaks inside the french hospitals. The same regulations are now put into force in most european countries, with the same result. They were some criticisms: "this is not evidence based ". We may accept this scientific point of view, but the obtained results seem demonstrate the validity of this strategy.

During the last decade the enumeration by molecular biology of both Legionella pneumophila and Legionella spp was made possible according the methodology initially developed and normalized in France and now worldwide disseminated. The results are taken into consideration for the diminution of the analyses performed on culture media. But the use of the culture method is always considered as the reference method for the official analyses. Until now there is no attempt for changing the limit values from CFU/L to genomic units $/ \mathrm{mL}$.

Some other sources of contamination were recently regulated like misting in public spaces, possible aerosolization through fountains, sprays, jacuzzis etc.. with the same requirement of regular analytical surveillance.

\section{Conclusion}

Confronted successively in 1998 and 2000 to mediatic legionellosis outbreaks, the french authorities, despite the absence of very scientifically sounded data, decided without delay to organize a mandatory active surveillance and to regulate both cooling towers and distribution networks inside public buildings for the control of Legionella aerosolization. The results may be considered as dramatic. The number of detected cases increased rapidly for being and remaining the highest rate of incidence in Europe despite a clear decrease during the last decade. The years 2010 and 2017, with very hot summers, experienced peaks of incidence which cannot be explained in absence of outbreaks. It may be hypothesized a climatic influence because the routinely performed controls did not show any difference with the 


\section{numbers of Legionnaires' disease cases in France}
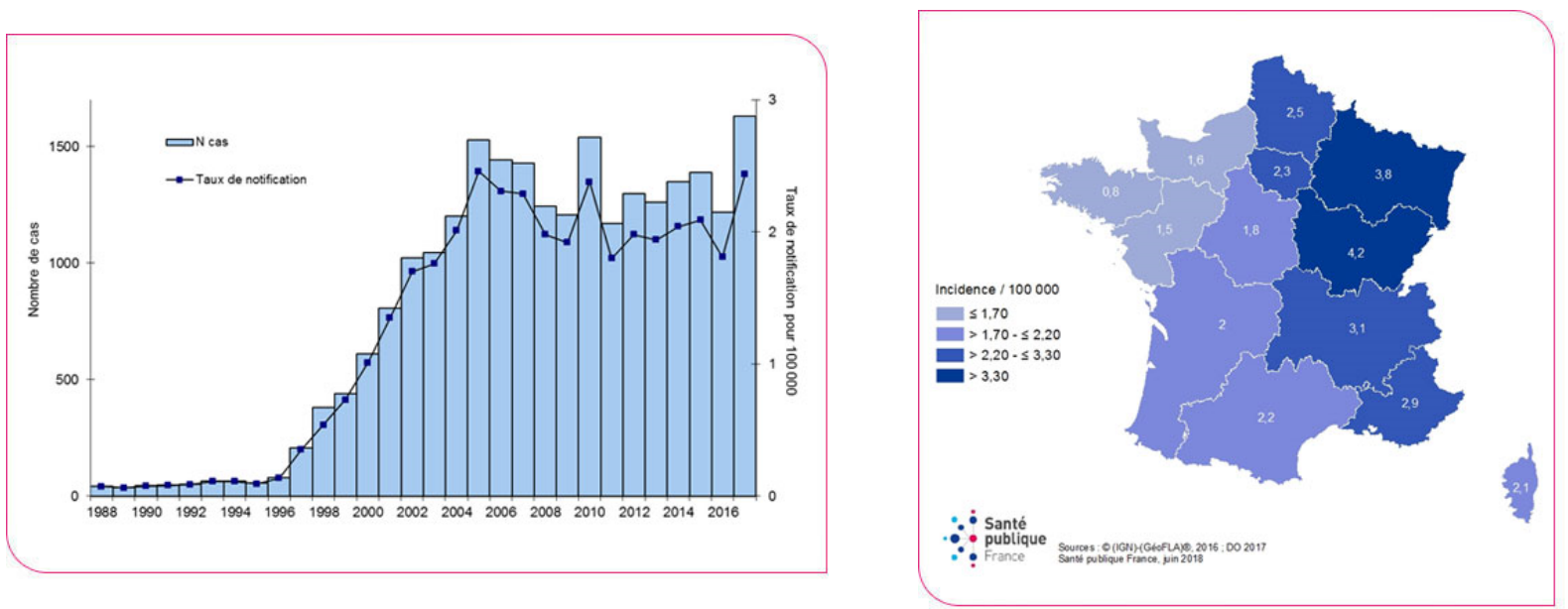

Figure 1. Epidemiological situation of legionellosis in France

\section{How to control the proliferation of Legionella in the distribution networks}

\section{1- Temperature measurement :}

-Hot water distribution system

introduced in a loop at around $60^{\circ} \mathrm{C}$ and

a return at $55^{\circ} \mathrm{C}$ : risk of burns; mitigation taps mandatory,

\section{-Cold water distribution network} maintained below $25^{\circ} \mathrm{C}$,

\section{2- Increase level of sample analysis}

Regular mandatory sampling of critical points,

\section{3-Preventive measures if unused network}

Figure 2. The compulsory measures for hot water distribution networks

other years. The summer of 2018 was also very hot, and we will see if the same phenomenon would occur.

Around half of these cases may not be attributed to a defined exposure, illustrating the difficulty, even a mandatory questionnaire and investigations performed by the local sanitary engineers. From the attributed cases, the percentage of those considered as Healthcare Associated Infections is continuously decreasing, leading to think the mandatory control of Legionella pneumophila produces satisfying results.

\section{Acknowledgement}

The author acknowledges all participants in the organization of Legionella surveillance and control in France for 20 years of continuous collaboration and especially the members of his research team in the Public Health and Environment Department of the School of Medicine of Nancy for their continuous support and deep involvement.

\section{References}

1. Decludt B, Guillotin L, Van Gastel B, Dubrou S, Jarraud S, et al. (1999) Foyer epidemique de legionelloses a Paris en juin 1998. Bull Epid Hebdo 4.

2. Weber W (2001) Paris' Pompidou hospital plagued by setbacks. Lancet 357: 130 [Crossref]

3. Campese C, Bitar D, Jarraud S, Maine C, Forey F, et al. (2011) Progress in the surveillance and control of Legionella infection in France 1998-2008. Int J Infect Dis 5: 30-37. [Crossref]

4. Nguyen TM, Ilef D, Jarraud S, Rouil L, Campese C, et al. (2006) A community-wide outbreak of Legionnaires disease linked to indutrial cooling towers -how far can contaminated aerosols spread? J Infect Dis. 193: 102-111. [Crossref]

5. Ministry of Health Editor (2005) Guide technique: l'eau dans les établissements de santé. 129.

6. Hartemann P, Hautemaniere A (2011) Legionellosis prevention in France. Bundesgesundheitsblatt Gesundheitsforschung Gesundheitsschutz 54: 724-727. [Crossref] 